\title{
AUTOLOGOUS CHONDROCYTE IMPLANTATION FOR KNEE FOCAL CARTILAGE DEFECTS: 3 YEARS' FOLLOW-UP AT THE UNIVERSITY MALAYA MEDICAL CENTRE
}

\author{
Abbas $A A^{1}$, Mohamad JA2, Lydia $A L^{3}$, Selvaratnam $L^{4}$, Razif $A^{1}, A b$-Rahim $S^{1}$,Kavitha $G^{1}$, Shilpa $P N^{5}, K_{\text {Kamarul }}{ }^{1}$ \\ 1 Tissue Engineering Group, NOCERAL, Department of Orthopaedic Surgery, Faculty of Medicine, University Malaya, \\ Kuala Lumpur, Malaysia \\ 2 Selangor Medical Centre, Shah Alam, Selangor, Malaysia \\ 3 Department of Rehabilitation Medicine, Faculty of Medicine, University Malaya, Kuala Lumpur, Malaysia \\ 4 School of Medicine and Health Sciences, Monash University Malaysia, Petaling Jaya, Selangor, Malaysia \\ 5 Virtis Bio Labs, Salem, India
}

Correspondence:

Dr. P. N. Shilpa

Virtis Bio labs

39, Eswari Gardens Annex,

Mannarpalayam

Kannankuruchi, Salem - 636008

Telephone : +91-9597926827

E-mail : shillpamail@gmail.com

\begin{abstract}
Autologous chondrocyte implantation $(\mathrm{ACl})$ is a widely accepted procedure for the treatment of large, fullthickness chondral defects involving various joints, but its use in developing countries is limited because of high cost and failure rates due to limited resources and support systems. Five patients (age $<45$ years) with focal cartilage defects received $\mathrm{ACl}$ at University of Malaya from 2006 to 2007 and followed up for 36 months. The average presubjective Knee Evaluation Forms (IKDC) improved from $38.44 \pm 6.29$ to $25.6 \pm 8.04$ postoperatively, the Oxford Knee Score (OKS) went from 25.6 \pm 8.04 to $13.96 \pm 1.63$ and the American Knee Society Score (AKSS) improved from $80 \pm 14.33$ to $92.96 \pm 5.82$ post-operatively. Thus improvements were seen in the IKDC and AKSS score but not in the OKS. Magnetic resonance images showed the presence of cartilage tissue filling in the lateral and medial patellar facet and medial femoral condyle in three patients. Failures were seen in two patients, both with patellar defects and over the age of 36 years. Treatment with autologous chondrocyte implantation for focal cartilage defect in lateral and medial patellar facet and medial femoral condyle showed early improvement which was maintained at 3 yrs follow-up. ACl provided satisfactory outcome in focal cartilage defects involving the femoral condyle.
\end{abstract}

Keywords: Knee, cartilage defect, autologous chondrocyte implantation, chondrocyte

\section{Introduction}

Focal chondral lesions pose a challenging problem to treat owing to loss of blood supply, low mitotic activity and immobility of articular chondrocyte (1). Although a number of surgical options are available to treat this increasingly common condition, each method has its own advantages and disadvantages (2). The aim of all cartilage repair methods is to reestablish functional properties of the damaged chondro-osseus unit. Debridement (3), drilling (4), and microfracturing of subchondral bone (5) have been advocated as treatment modalities. More recently, autogenous osteochondral plugs, osteochondral allografts, and autologous chondrocyte implantation $(\mathrm{ACl})$ have been used. These techniques have been reported to have good short- to mid-term results (6).

$\mathrm{ACl}$ is a two-stage procedure involving the harvesting of cartilage from a non-weight-bearing area of the joint, followed by chondrocyte isolation and culture. Cells are then harvested from culture and reimplanted into defective sites. These cells undergo further proliferation and de-differentiation within the damaged area (7). Newly formed tissue within the treated area will mature over time, developing into more organized tissue that mimics the surrounding native cartilage (hyaline-like cartilage) (8). Because of the novelty of this treatment, $\mathrm{ACl}$ continues to be evaluated for its consistency and reproducibility in 
producing good clinical outcomes (8-11). The durability of repair and cost effectiveness is also being questioned (12). Despite the popularity of the technique, the factors influencing the functional outcome after $\mathrm{ACl}$ are still poorly understood. Furthermore, studies using $\mathrm{ACl}$ in developing countries that have restricted resources are limited. This report describes the results of a preliminary cohort study conducted at the University of Malaya to determine the clinical outcome of five patients aged below 45 years who were treated for focal cartilage defect using $\mathrm{ACl}$.

\section{Materials and methods}

\section{Patients}

Five patients aged below 45 years underwent $\mathrm{ACl}$ for treatment of focal knee cartilage defects between 2006 and 2007. Approval to conduct the study was given by the medical ethics committee (Medical Ethics Committee Ref No: 553.37), University of Malaya. In accordance with Malaysian law, patients were informed of the nature of the study and provided written consent. Patients' histories, detailing the mechanism of injury, onset, and symptoms as well as prior treatments were recorded for preoperative assessment. Patients with cartilage defects down to, but not through, the subchondral bone, on a load-bearing surface of the femoral condyle or the patellar facet were recruited for treatment. The patients presented with a variety of complaints, including localized pain, swelling, and retropatellar crepitus. Preoperative assessments included use of the 2000 IKDC Subjective Knee Evaluation Forms (13), the Oxford Knee Score (OKS) (14), the American Knee Society Score (AKSS) (15), anteroposterior and lateral knee radiographs and magnetic resonance imaging (MRI).The $\mathrm{ACl}$ technique was performed as described by Brittberg et al. (16).

\section{Isolation and Culture of Chondrocytes}

Patients underwent diagnostic arthroscopy of the knee under general anaesthesia. A tourniquet-controlled, bloodless field provided good visualization of the defect(s) sites as well as the areas suitable for cartilage harvesting. Approximately 2-3 g of cartilage tissue was removed using a surgical punch. The harvested tissue was placed in a sterile container containing phosphate-buffered saline supplemented with penicillin and streptomycin. The tissue samples were sent to the laboratory for further processing within 4 hours of retrieval.

Chondrocytes were cultured in an ISO-certified class 1000 clean laboratory located in the Faculty of Medicine, University of Malaya. Harvested tissue was minced before being digested in collagenase-type II solution for $24 \mathrm{~h}$. The following day, the suspension was centrifuged at 1800 rpm for $10 \mathrm{~min}$ to produce the cell pellet. The pellet was resuspended in DMEM/F-12 growth medium at a ratio of $1: 1$, supplemented with $10 \%$ fetal bovine serum and 25 $\mu \mathrm{g} / \mathrm{ml}$ of ascorbic acid. Cultures were stored in $5 \% \mathrm{CO}_{2}$ and $98 \%$ humidity at $37^{\circ} \mathrm{C}$. The medium was replaced every 3-4 days. Observation continued until $80 \%$ cell confluence was reached, after which the culture was trypsinized to detach the cells from the plastic surfaces for further passage. Cell cultures were expanded up to the third passage to allow the recovery of $2-5 \times 10^{6}$ cells, which took approximately 4-6 weeks. Cell viability was analysed using trypan blue technique (17).

\section{Implantation}

Prophylactic antibiotics (Cefuroxime sodium, $750 \mathrm{mg}$ ) were given intravenously in three doses over 24 hours during and after surgery. Cell implantation was performed as described by Brittberg et al. (16). A medial or lateral parapatellar arthrotomy was performed in a tourniquet-controlled, bloodless field. To ensure that only healthy tissue remained in the defect, the margin of the defect was excised whilst the base was scraped using a scalpel to remove tissue remnant. Care was taken to ensure that the subchondral plate was not penetrated. A periosteal flap, identical in shape and size with the lesion area, was harvested from the medial aspect of the proximal tibia, or the supracondylar region of the femur of the affected knee. This flap was used to cover the cartilage defect, with the cambium layer facing the subchondral bone in the defect area. The flap was sutured to the surrounding rim of the normal cartilage with interrupted 6-0 Vicryl ${ }^{\circledR}$ sutures, leaving an opening in the upper part of the defect for insertion of cultured chondrocytes. The intervals between the sutures were sealed with fibrin glue, and the patch was tested for watertightness by injecting saline into the defect and checking for leakage. More than $48 \times 10^{6}$ cultured chondrocytes (four vials) were administered beneath the periosteal flap, and the opening was closed with suture and fibrin glue. The joint capsule, retinaculum layer and skin were sutured in separate layers. In the case of patellar maltracking, the lateral parapatellar joint capsule was not repaired. The knee was covered with a small elastic bandage.

\section{Postoperative Protocols}

Continuous passive motion was initiated within 6 hours postoperatively, with the range of flexion limited to $30^{\circ}$. This was continued until patients were able to mobilize the knee independently. Quadriceps strengthening exercises were encouraged during the recovery period. Active movement of the knee without weight-bearing was initiated 2-3 days after surgery. Patients were discharged with a protective knee brace that limited flexion to $45^{\circ}$. Once discharged, patients attended outpatient physiotherapy twice weekly, initially for 12 weeks and then, subject to their progress, once weekly. Weightbearing was gradually increase, along with knee flexion increased to full extension, with isometric quadriceps training during the first 8 weeks after surgery. 


\section{Follow-up}

Patients were evaluated at 3, 6, 12, 24 and 36 months postoperatively. Evaluation with the 2000 IKDC Subjective Knee Evaluation Forms, OKS and the AKSS was performed at 3-monthly intervals, while MRI assessments were performed at 6 months postoperatively. The AKSS score included surgeons' reports of patients' symptoms, impairment (clinical examination findings) and disability measured with the AKSS before and 1 year after surgery. The AKSS generates a knee score from 0 to 100, based on symptoms and impairment, and a function score from 0 to 100 calculated from the answers to questions on disability. Higher scores indicate lesser symptom severity, impairment or disability. The OKS, a joint-specific instrument, consists of 12 questions to assess pain and physical disability on a five-point Likert scale (1 representing no pain or disability, to 5 representing extreme pain or disability), which yields a single score (i.e. sum of all individual items) ranging from a best functional outcome of 12 to a worst functional outcome of 60 .

\section{Results}

The five patients (two men, three women) were aged 25-45 years (mean, 37.2 years). The average defect size was $8 \mathrm{~cm}^{2}$ (range: $4.0-12.0 \mathrm{~cm}^{2}$ ). Demographic data for all five patients who underwent $\mathrm{ACl}$ are shown in Table 1.

The IKDC, OKS and AKSS scores increased in three patients after $\mathrm{ACl}$ as early as 3 months postoperatively and continued to improve up to 24 months and remained at the same level up to 36 months. The mean age of patients who responded well to $\mathrm{ACl}$ was 36 years, while that of patients who had a poorer outcome was 39.5years. The average preoperative and postoperative IKDC, OKS and AKSS scores of patient are shown in Table 2.
It can be argued that while the high scores at 3 months may be controversial since patients are not fully ambulating, the data presented here is outcome perceived by the patients and not really of the objective physical outcome. There was little or no pain and satisfaction was high in all patients at 3 months. This may have explained the reason for the high score observed at 3 months follow-up.

At 36 months' follow-up, three patients had excellent results, while in two patients knee function was found to be poor, as reflected in decreased IKDC, OKS and AKSS scores (Figures $1,2,3)$. MRI of three patients with good knee functional scores showed that the defective area was completely filled with reparative tissue, highlighted with hyperintense signals. In the two patients with a poor clinical response, the area surrounding the repaired cartilage had lower density. In the three patients with good clinical response i.e. OKSS of less than 15 and IKDC of more than 50, an increase of 2-3 $\mathrm{mm}$ in the depth of the repair tissue was observed over time, being most remarkable 24-36 months after surgery (Figure 4).

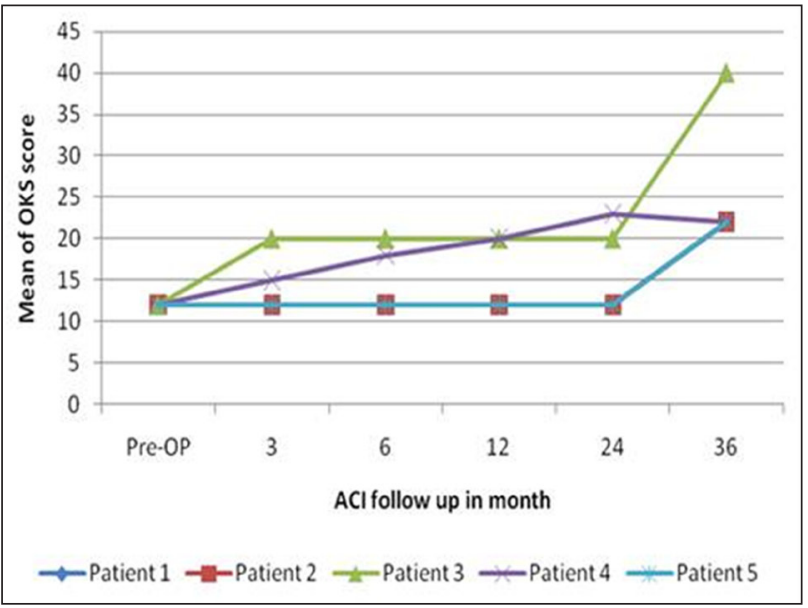

Figure 1: Mean AKSS score preoperatively and after 3 years' follow-up. Patients 1 and 2 have same mean score.

Table 1: Demographic data of patient receiving ACl to treat knee focal cartilage defect

\begin{tabular}{|c|c|c|c|c|c|c|}
\hline \multirow[t]{2}{*}{ Case } & \multirow[t]{2}{*}{ Gender } & \multirow[t]{2}{*}{ Age } & \multicolumn{2}{|l|}{ Cartilage lesion } & \multirow[t]{2}{*}{ Mechanism of Injury } & \multirow[t]{2}{*}{ Outcome } \\
\hline & & & Site & Size $(\mathrm{mm})$ & & \\
\hline 1. & Male & 45 & Lateral and medial patellar facet & 8 & Patellar maltracking & Successful \\
\hline 2. & Female & 34 & Medial femoral condyle & 4 & Sports & Successful \\
\hline 3. & Male & 29 & Medial femoral condyle & 4 & Sports & Successful \\
\hline 4. & Female & 33 & Lateral patellar facet & 4 & Patellar maltracking & Failure \\
\hline 5. & Female & 45 & Trochlea and patella & 4 & Trauma & Failure \\
\hline
\end{tabular}

Table 2: Outcome of OKS, IKDC, AKSS scoring of patient receiving ACI to treat knee focal cartilage defect during three years followup.

\begin{tabular}{lllllll}
\hline Score & Preoperatively & 3 months & 6 months & 12 months & 24 months & 36 months \\
\hline OKS & $25.6 \pm 8.0$ & $15.8 \pm 5.3$ & $15.2 \pm 4.3$ & $14.8 \pm 3.8$ & $12 \pm 3.4$ & $14.2 \pm 3.12$ \\
IKDC & $38.44 \pm 6.2$ & $62.4 \pm 18.4$ & $64.8 \pm 20.0$ & $67.4 \pm 22.4$ & $83.3 \pm 23.5$ & $83.35 \pm 5.7$ \\
AKSS & $80 \pm 14.3$ & $86.6 \pm 16.4$ & $88.6 \pm 15.6$ & $89.6 \pm 14.2$ & $100 \pm 14.2$ & $100 \pm 12.7$ \\
\hline
\end{tabular}




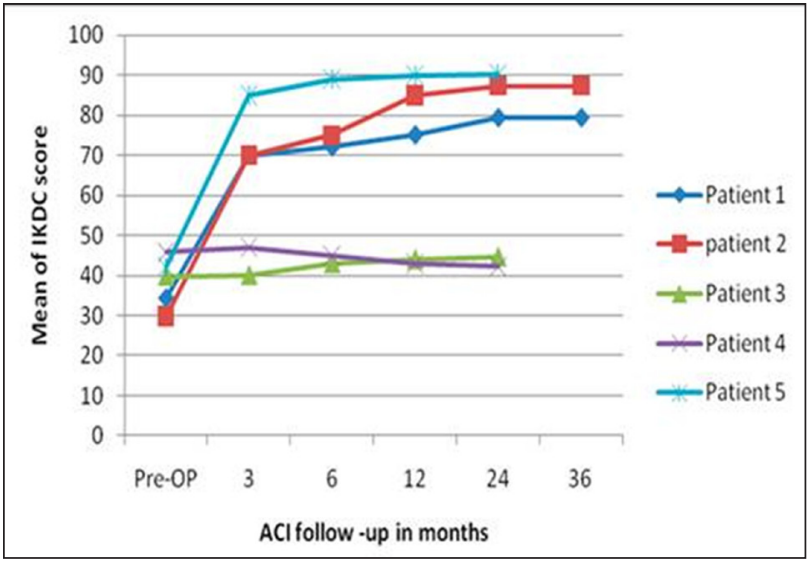

Figure 2: Mean IKDC score preoperatively and after 3 years' follow-up.



Figure 3: Mean OKS score preoperatively and three years' follow-up. Patient 1, 2 and 5 have same mean score

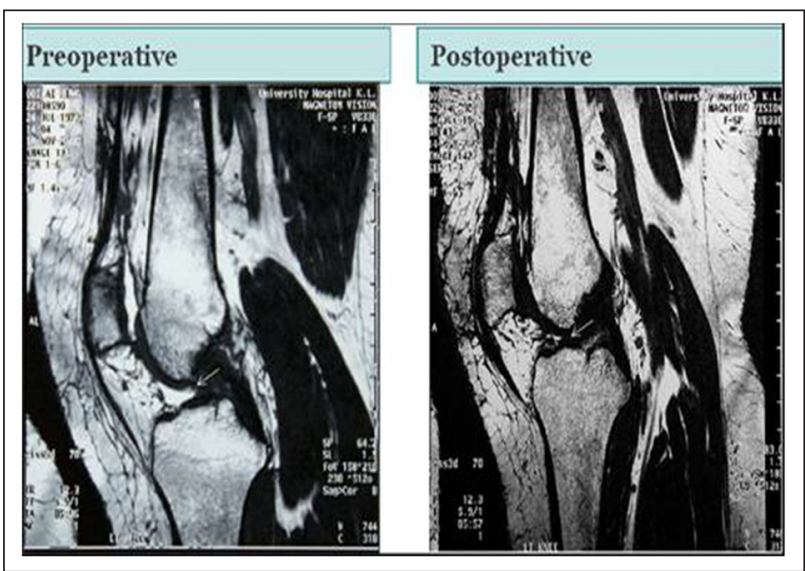

Figure 4: Magnetic resonance images obtained 3 year after autologous chondrocyte implantation preoperatively and postoperatively

\section{Discussion}

An overall improvement in patient functional scores for up to 3 years has been observed in patients who underwent $\mathrm{ACl}$. This preliminary study on the use of $\mathrm{ACl}$ in selected patients reveals that this technique can be used successfully. There appears to be a dramatic improvement in patient satisfaction and knee function within 3 months following surgery (38-60\%) and continue to increase over time. This finding was similar to that reported by Micheli et al. which demonstrated an average improvement of up to $84 \%$ in patients who underwent $\mathrm{ACl}$ after $2-3$ years (10).

The major limitation of this report is the small number patients. However, this report does provide preliminary evidence advocating its use for continued recruitment for patients in a large scale study. The results also suggest that patients with a patellar defect: may not be suitable candidates. Similar outcomes were also observed in previous published results (8).

Although in a number of studies, it has been reported that low number of cells implanted in cartilage defects may have contributed to the failures observed, however it is unlikely to be the case within the present study. In patients recruited for this study, at least $48 \times 10^{6}$ cells ( 4 vials) were administered to each patient, which was similar if not higher than those reported in previous studies (18). Although the optimal number of required cells has not yet been determined, high cell densities seem to be desirable and recommended by most authors (19). In several studies, the number of chondrocytes seeded in the initial defects appears to be linearly correlated to the biosynthetic activity required for cartilage restoration (20). The higher numbers of cells used in this study as compared to those used in conventional studies suggests that the use of higher number of cells would not have influenced the outcome. Instead, the site of injury may be the determinant factor which may influence the outcome of the repair process.

As with previous reports, patient age has been found to be a confounding factor for the success in $\mathrm{ACl}$ (11). In our study, patients under 36 years showed better improvement in all scores than those exceeding 36 years. This may have resulted from the use of ageing chondrocytes, related to the advanced age of the donors. It has been suggested that the ageing chondrocytes have limited regenerative ability by producing limited extracellular matrix hence, resulting in poor repair outcomes (5). Nevertheless, even in patients with degenerative cartilage lesions, the use of $\mathrm{ACl}$ may be feasible albeit with limited success (21-23). However, this issue remains controversial and requires substantial evidence before it can be routinely advocated.

Of the many available methods used to access cartilage restoration, many studies have advocated the use of MRI, mainly because it is non invasive, reliable and, allows comparative analyses to be made between the pre- and post-operative conditions (24). In patients who were successful at 3 years, $2-3 \mathrm{~mm}$ filling of what appears to be cartilage tissue i.e. not bone and not synovial due to the apparent density, within the medial femoral condyle and patella defects were observed. It is not unexpected that patients with patellofemoral defects are more likely 
to fail, as lesions of the patellofemoral joint have always been more demanding and more difficult to treat than femoral condyle lesions (25). This can be explained by the more complex pathogenesis involved resulting from the complicated anatomical malfunction which causes patellofemoral malalignment, maltracking and ultimately the instability of the patella. In a report published by Peterson et al. (26) involving 94 patients who underwent $\mathrm{ACl}$ and had been followed up for 2-9 years, good or excellent clinical outcomes were achieved in $76 \%$ of the patients with isolated condylar lesions while the worst outcome was seen in those who had multiple defects or trochlear lesions and patella. Peterson et al. (9) also showed that lesions of patellar femoral joint were more difficult to treat than femoral condyle lesions.

Based on available literature, it appears that the repair process following implantation of autologous chondrocytes may be the result of multiple biological and mechanistic cellular functions acting in concert (27). It has been suggested that among those most commonly described, the repopulation of implanted chondrocytes within the defect sites appears to be the more accepted notion (28). Once adapted into the surrounding environment, these cells are said to produce extracellular matrix which inherently provides the repair tissue which is observed at the end of the repair process. There is however, no clear evidence to support this assumption as studies have demonstrated that these implanted cells do not proliferate when contained within the defect site and up to $87 \%$ of these cells undergo apoptosis within 4 weeks (29-31). It has also been proposed that it is not the transplanted cells which result in the repair but the use of periosteum as a cover which promotes tissue healing (32). However, this too has been refuted as studies involving rabbits have shown that using periosteum alone does not result in repair of the cartilage tissues (32-35). In addition, studies using synthetic patches instead of periosteum in patients also resulted in the regeneration of damaged cartilage (36).

While it is not clear as to what causes the cartilage defect to undergo repair when $\mathrm{ACl}$ is used, our result showed apparent improvement in tissue repair as observed in many clinical and laboratory studies $(36,37)$. Further studies to elucidate the causes and mechanisms leading to the regenerative process should therefore be conducted in larger and more robust experiments, with hopes that better understanding of $\mathrm{ACl}$ can be achieved, therefore producing superior results in clinical use.

\section{Conclusion}

The present case study provides support to use $\mathrm{ACl}$ in selected patients with focal cartilage defects involving the femoral condyle. The use of $\mathrm{ACl}$ in patients above the age of 36 or those with patellar defects are not encouraged, but need to be supported by larger scale studies.

\section{References}

1. Minas T. Autologous chondrocyte implantation for focal chondral defects of the knee. Clin Orthop Relat Res 2001; 391:349-361.

2. Brian JC, Cecilia PG, Robert CG. Surgical management of articular cartilage defects in the knee. J Bone Joint Surg 2009; 91:1778-1790.

3. Hubbard MJ. Articular debridement versus washout for degeneration of the medial femoral condyle: a five-year study. J Bone Joint Surg Br 1996; 78:217-219.

4. Gilbert JE. Current treatment options for the restoration of articular cartilage. Am J Knee Surg 1998; 11:42-46.

5. Blevins FT, Steadman JR, Rodrigo JJ et al. Treatment of articular cartilage defects in athletes: an analysis of functional outcome and lesion appearance. Orthopedics 1998; 21:761-768.

6. Hunziker EB. Articular cartilage repair: basic science and clinical progress. A review of the current status and prospects. Osteoarthr Cartil 2002; 10:432-463.

7. Brittberg M, Winalski CS. Evaluation of Cartilage Injuries and Repair. J Bone Joint Surg Am 2003; 85:5869.

7. Bernhard JT, lain WM, Tomoki T, et al. Autologous chondrocyte implantation in knee Joint: MR Imaging and histologic Features at 1-year Follow-up. Radiology 2005; 234:501-508.

8. Peterson L, Vasiliadis SH, Brittberg M et al. Autologous chondrocyte implantation:A long-term follow-up. Am J Sports Med 2010; 38:1117-1124.

9. Micheli LJ, Browne JE, Erggelet $\mathrm{C}$ et al. Autologous chondrocyte implantation of the knee: multicenter experience a minimum 3 year follow-up. Clin. J Sport Med 2001; 11:223-228.

10. Niemeyer P, Kostler W, Salzmann GM et al. Autologous chondrocyte implantation for treatment of focal cartilage defects in patients age 40 Years and older. A matched-pair analysis with 2-Year follow-up. Am J Sports Med 2010; 38:2410-2416.

11 Clar C, Cummins E, Mclntyre L et al. Clinical and cost-effectiveness of autologous chondrocyte implantation for cartilage defects in knee joints: systematic review and economic evaluation. Health Technol Assess. 2005; 9(47):1-82.

12. Irrgang JJ, Anderson AF, Boland AL et al. Development and validation of the intenational knee documentation committee subjective knee form. Am J Sports Med. 2001; 29(5):600-613.

13. Dawson J, Fitzpatrick R, Murray D et al. Questionnaire on the perceptions of patients about total knee replacement. J Bone Joint Surg Br. 1998; 80(1): 63-69.

14. Insall JN, Dorr L D, Scott RD, et al. Rationale of the knee society clinical Rating System. Clin Orthop 1989; 248:13-14.

15. Brittberg M, Lindahl A, Nilsson A et al. Treatment of deep cartilage defects in the knee with autologous 
chondrocyte transplantation. N Engl J Med 1994; 331:889-895.

16. Morgan SJ, Darling DS. Animal cell culture: A practical approach. 2nd ed. IRI press; 1992.

17. Kim SJ, Chang CH, Jang JD et al. A multi-center study of repairing articular cartilage defects of the knee using cultured autologous chondrocytes. Zhongguo Zuzhi Gongcheng Yanjiu yu Linchuang Kangfu 2009; 13:6418-6422.

18. LeBaron RG, Athanasiou KA. Ex vivo synthesis of articular cartilage. Biomaterials 2000; 21:2575-2587.

19. Chen AC, Nagrampa JP, Schinagl RM et al. Chondrocyte transplantation to articular cartilage explants in vitro. J Orthop Res. 1994; 15(6):791-802.

20. Kreuz PC, Muller S, Ossendorf C et al. Treatment of focal degenerative cartilage defects with polymerbased autologous chondrocyte grafts: four-year clinical results. Arthritis Res Ther 2009; 11:R33.

21. Parsch D, Brummendorf TH, Richter $\mathrm{W}$ et al. Replicative aging of human articular chondrocytes during ex vivo expansion. Arthritis Rheum. 2002; 46(11):2911-2916.

22. Mithoefer K, Williams RJ, Warren RF et al. The microfracture technique for the treatment of articular cartilage lesions in the knee: a prospective cohort study. J Bone Joint Surg Am 2005; 87:19111920.

23. Roberts S, McCall IW, Darby AJ et al. Autologous chondrocyte implantation for cartilage repair: monitoring its success by magnetic resonance imaging and histology. Arthritis Res Ther 2003; 5:60-73.

24. Peterson L, Brittberg M, Kiviranta I et al. Autologous chondrocyte transplantation: biomechanics and longterm durability. Am J Sports Med 2002; 30:2-12.

25. Peterson L, Minas T, Brittberg M, et al. Two-to9-year outcome after autologous chondrocyte transplantation of the knee. Clin Orthop 2000; 374:212-234.

26. Stoltz JF, de Isla N, Huselstein Cet al. Mechanobiology and cartilage engineering: The underlying pathophysiological phenomena. Biorheology 2006; 43:171-180.
27. Min BH, Woo JI, Kim WH et al. The fate of implanted autologous chondrocytes in regenerated articular cartilage. Proc. IMechE Part H: J. Engineering in Medicine 2007; 221:461-465.

28. Ostrander RV,Goomer RS, Tontz WL et al. Donor cell fate in tissue engineering for articular cartilage repair. Clin Orthop Relat Res 2001; 389:228-372.

29. Mierisch CM, Wilson HA, Turner MA et al. Chondrocyte transplantation into articular cartilage defects with use of calcium alginate: the fate of the cells. J Bone Joint Surg Am 2003; 85:1757-1767.

30. Zeifang F, Oberle D, Nierhoff $C$ et al. Autologous chondrocyte implantation using the original periosteum-cover technique versus matrix-associated autologous chondrocyte implantation: a randomized clinical trial. Am J Sports Med 2010; 38:924-933.

31. Peterson L, Menche D,Grande D et al. Chondrocyte transplantation-an experimental model in rabbit. In:Transactions from the $30^{\text {th }}$ Annual Orthopedic Research Society, Atlanta, February7-9, 1984. Palatin,III.: Orthopedic Research Society: 218.

32. Brittberg $M$, Nilsson $A$, Peterson $L$ et al. Healing of Injured rabbit articular cartilage after transplantation with autologously isolated and cultured chondrocytes. In:Abstracts of the Bat Sheva Seminars on Methods Used in Research on Cartilaginous Tissues, Tel AVIV,March 26, 1989.Vol.1, Ginnosar, 91:1778-1790.

33. Grande DA, Pitman MI, Peterson Let al. The repair of experimentally produced defects in rabbit articular cartilage by autologous chondrocyte transplantation. J Orthop Res 1998; 7:208-218.

34. Kreuz PC, Muller S, Ossendorf C et al. Treatment of focal degenerative cartilage defects with polymerbased autologous chondrocyte grafts: four-year clinical results. Arthritis Res Ther 2009; 11:R33.

35. Kamarul T, Ab-Rahim S, Tumin M et al. A preliminary study of the effects of glucosamine sulphate and chondroitin sulphate on surgically treated and untreated focal cartilage damage cells and material. Eur Cells Mater 2011; 121:259-271.

36. Bentley G, Biant LC, Carrington RW et al. A prospective, randomized comparison of autologous chondrocyte implantation versus mosaicplasty for osteochondral defects in knee. J Bone Joint Surg $\mathrm{Br}$ 2003; 85:223-230. 